\title{
Prospects of development of a method for separate estimation of the contributions of corrosion products and inhibitor films to the total protective effect
}

\author{
R. V. Kashkovskiy \\ Gazprom VNIIGAZ, LLC, Razvilka, Leninsky Rayon, Moscow Region, \\ 142717 Russian Federation.E-mail: roman.kashkovskiy@gmail.com
}

\begin{abstract}
This paper offers a modification of a method for separate estimation of the contributions of corrosion products and inhibitor films to the total protective effect. The method is based on the consideration of changes in thickness of the corrosion product film due to the inhibitor effect and film dissolution. A comparison of the original and modified methods is based on experimental results.
\end{abstract}

Key words: corrosion, corrosion products, inhibitor, hydrogen sulfide, steel, amine.

Received: July 17, 2013.

doi: $\underline{10.17675 / 2305-6894-2013-2-4-269-276}$

\section{Introduction}

It is known that the film of corrosion products (CP) that starts to form on the metal surface directly at the instant of its contact with a corrosive environment often has certain protective properties and leads to a significant decrease in the corrosion rate $[1,2]$, especially at the initial stage of exposure. In the case of corrosion inhibition, the inhibitor is adsorbed on the CP film. In this case, corrosion hindrance is usually attributed only to the presence of a phase inhibitor layer on the metal surface, whereas the protective CP film is not taken into account. This assumption leads to a significant overestimation of the inhibitor protective effect, and the more protective CP film is formed on a metal, the greater the error. However, it is only recently that this topic became the subject of studies in a number of papers [3-5], in spite of its scientific and practical significance.

A simple and informative method for estimating the contributions of planar components of a surface film to the total protective effect is offered in paper [3]. The method is based on the analysis of corrosion and inhibition kinetics. The contribution of corrosion product films $\left(Z_{\mathrm{CP}}\right)$ to the total protective effect of a " $\mathrm{CP}$-inhibitor" surface system $\left(Z_{\text {total }}\right)$ is estimated by the formula:

$$
Z_{C P, \tau}=\frac{K_{b g, \tau \rightarrow 0}-K_{b g, \tau}}{K_{b g, \tau \rightarrow 0}},
$$

where $K_{b g, \tau}$ is the corrosion rate in the background environment at time $\tau$. 
It is necessary to correlate $Z_{C P}$ values with the thickness of the $C P$ film formed in the background environment to calculate $Z_{C P}$ in the inhibited environment. The thickness of a $\mathrm{CP}$ film is directly proportional to the specific weight loss of the sample in case of uniform growth of the CP film on the whole surface of corroded metal, i.e., $h=$ const $\times \Delta m_{s p}$, where $h$ is the CP film thickness and $\Delta m_{s p}$ is the specific weight loss of the sample. Further, we should simultaneously plot in the diagram the kinetics of specific weight losses of the metal in the background and inhibited environments and $Z_{C P}$ calculated using Eq. (1) based on corrosion data in the background environment. After that it is possible to estimate $Z_{C P}$ in the course of corrosion inhibition at any time $(\tau)$ in the following way (Fig. 1). The abscissa of the intersection point of curve 1 and a perpendicular drawn from point $A$ to axis OY determine the time $\tau_{A}$ when the sample in the background solution (curve 1) had the same specific weight loss and, hence, the same CP film thickness as in point A in case of corrosion inhibition. Moreover, it is possible to calculate the protective effect of the $\mathrm{CP}$ film at time $\tau_{A}\left(Z_{A}\right.$ point, Fig. 1) from the $Z_{C P}-\tau$ relationship (curve 3 ). Thus, one can estimate the kinetics of $Z_{C P}$ variation by calculating $Z_{C P}$ in the inhibited environment at various times $\tau$.

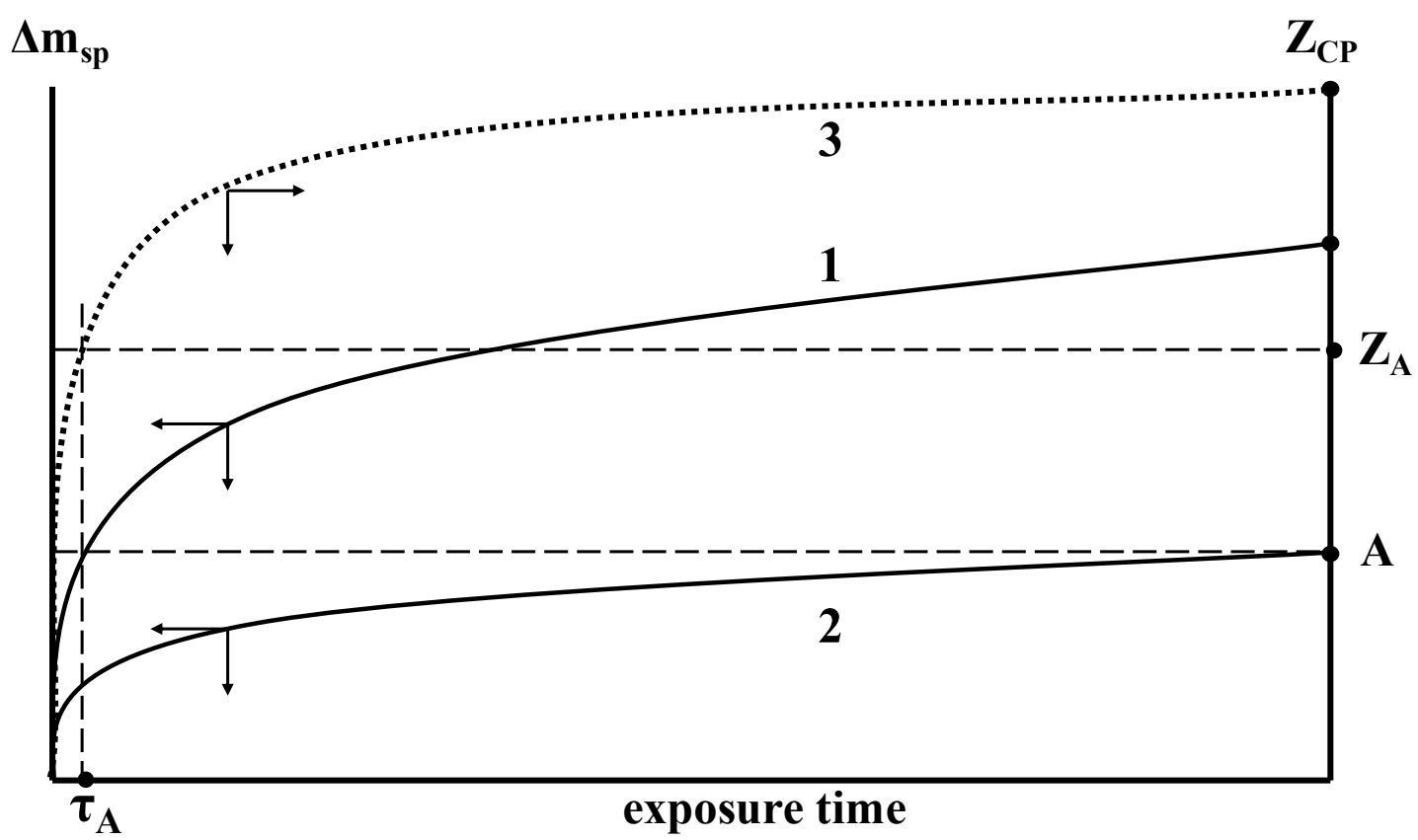

Figure 1. Kinetics of specific weight loss of metal sample in background (1) and inhibited (2) environment and $Z_{C P}(3)$.

The proposed method does not allow us to deduce directly the analytical correlation between $Z_{i n}$ value and $K$ kinetics in the background and inhibited environment. However, the formula for the calculation of the total protective effect from [1] $\left(Z_{\text {total }}\right)$ remains correct:

$$
Z_{\text {total }, \tau}=\frac{K_{b g, \tau \rightarrow 0}-K_{i n, \tau}}{K_{b g, \tau \rightarrow 0}} .
$$


The next equation follows from the previous one

$$
Z_{\text {in, } \tau}=Z_{\text {total }, \tau}-Z_{C P, \tau} .
$$

The latter equation does not contradict the concept underlying this method since $Z_{\text {total }}$ represents the total protective effect of a "CP film - inhibitor" system, including redistribution of their contributions due to a reduction in CP film thickness. One can determine the kinetics of variation in the contributions by calculating $Z_{\text {total }}$ by Eq. (2) and then $Z_{\text {in }}$ by Eq. (3) for various times $\tau$.

Theoretical bases of the modified method have been discussed in detail earlier $[4,5]$ and consequently are not described in this paper.

Nevertheless, assuming that an inhibitor film has no influence on a CP film (additive case), it is necessary to consider $\mathrm{CP}$ film thickness reduction during the decrease in corrosion rate caused by the inhibitor and also dissolution of the film that leads to changes in its protective properties in inhibited environment in comparison with a background film.

Therefore, as it was discussed in [5], consideration of CP film dissolution in a liquid phase is a possible improvement in the modified method. This value can be obtained by measuring the concentration of metal ions in the test solution after the experiment (iron ions in the case of steel). In this case, the CP thickness is directly proportional to the specific weight loss of the sample minus the weight of the metal transferred to the solution during film dissolution, i.e., $h=$ const $\times \Delta m_{s p}-C$, where $C$ is a parameter considering dissolution of the CP film. Further it is necessary to plot the specific weight loss of the metal less the quantity of dissolved $\mathrm{CP}$ instead of simple $\Delta m_{s p}$ on the " $\Delta m_{s p}\left(Z_{C P}\right)-\tau$ " graph (Fig. 1). The latter value, in turn, more precisely characterizes $Z_{C P}$ because it expresses the total amount of corrosion products adsorbed directly on the metal surface.

The modified method for estimation of the contributions to the total protective effect is presented in this paper using the example of inhibition of hydrogen sulfide corrosion (HSC) with consideration for reduction in the CP film thickness due to the inhibitor effect and film dissolution. The purpose of the paper is to compare the results provided by the original method (without consideration of CP film dissolution) and the modified method.

\section{Experimental}

Tributylamine (TBA) that is an efficient inhibitor of steel HSC in liquid and gas phases [4] was studied in the paper. The corrosion rate in the background and inhibited environments was determined by the Linear Polarization Resistance method using a two-electrode steel sensor ( $S=5 \mathrm{~cm}^{2}$ each) and a Monicor-2 corrosion rate indicator (IPTER production, Russia) under $10 \mathrm{mV}$ electrode polarization under stirred solution conditions (300 rpm). Steel electrodes made of low-carbon St3 steel were ground by emery paper, polished with diamond paste, degreased with acetone and kept in a desiccator for not less than 1 hour before each test.

A standard NACE medium $\left(0.25 \mathrm{~g} / \mathrm{L} \mathrm{CH}_{3} \mathrm{COOH}, 0.5 \mathrm{~g} / \mathrm{L} \mathrm{NaCl}, \mathrm{pH} 3.6\right)$ was used as the corrosive electrolyte simulating formation water at gas condensate fields. Gaseous $\mathrm{H}_{2} \mathrm{~S}$ 
was permanently purged through the electrolyte during the test. The amine was fed directly into the liquid phase.

Experiments were carried out in $0.5 \mathrm{~L}$ glass vessels at room temperature under normal atmosphere pressure. The duration of each test was 6 hours. The concentrations of Fe ions in the solutions after the tests were determined by colorimetry (method 2, GOST 4011-72 [6]). The mass of dissolved iron was calculated with consideration for the component composition of St3 steel [7].

\section{Results and Discussion}

Figure 2 shows HSC kinetics in the liquid $\left(\mathrm{C}_{\mathrm{H}_{2} \mathrm{~S}} \approx 3 \mathrm{~g} / \mathrm{L}\right)$ without and with TBA $(50,500$, $2000 \mathrm{mg} / \mathrm{L}$ ). All the correlations obtained have an inversely logarithmic characteristic which is an evidence of permanent protective film formation. Addition of TBA to the test solution considerably reduces corrosion rate that undoubtedly leads to a decrease in the CP film thickness in inhibited environment in comparison with the background solution.

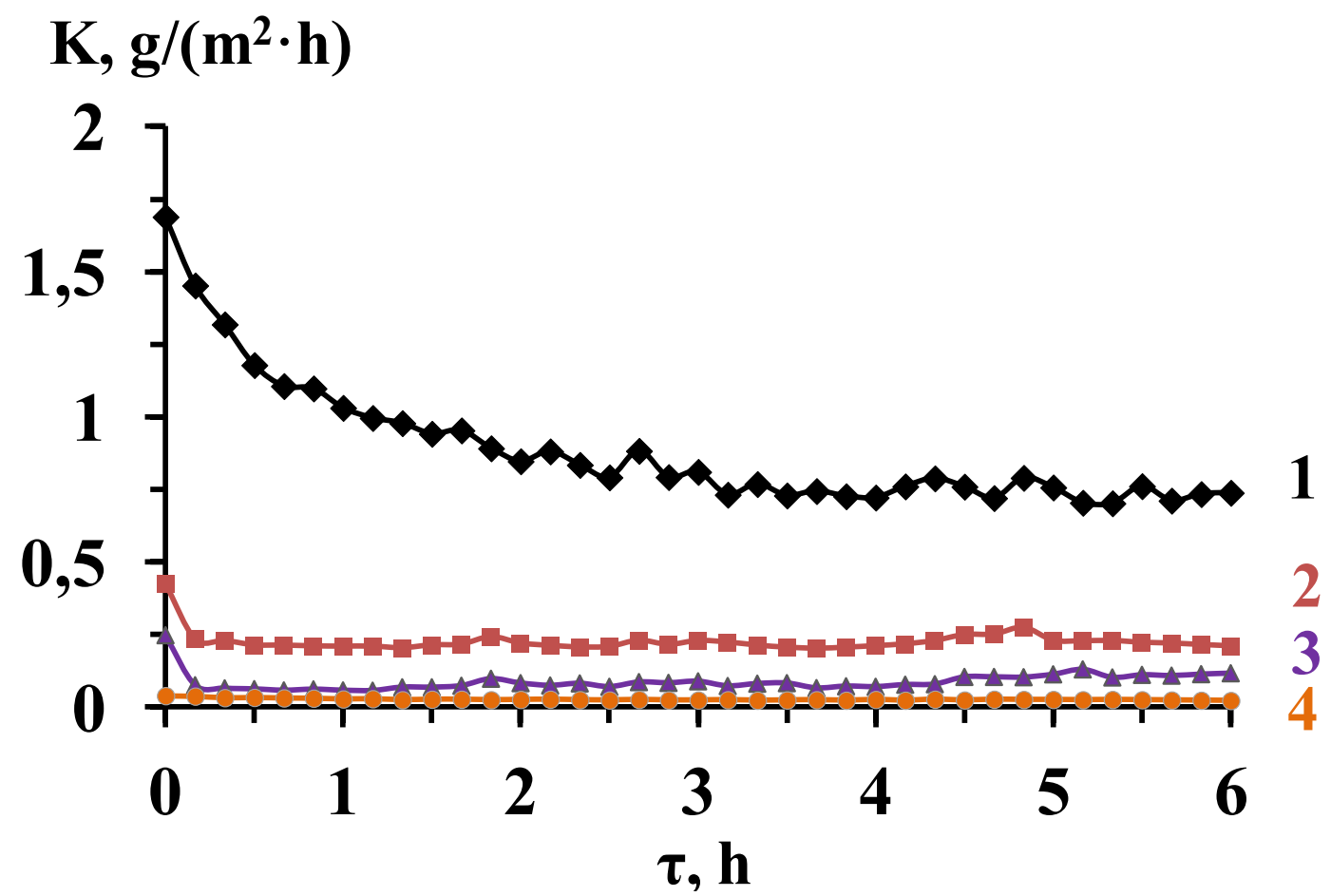

Figure 2. Correlation of St3 HSC rate over time in the background electrolyte (1) and in the presence of $50(2), 500(3)$ and $2000 \mathrm{mg} / \mathrm{L}$ of TBA (4).

The total content of iron in the tested solutions was measured during the experiments. The experimental data obtained are shown in Fig. 3. 


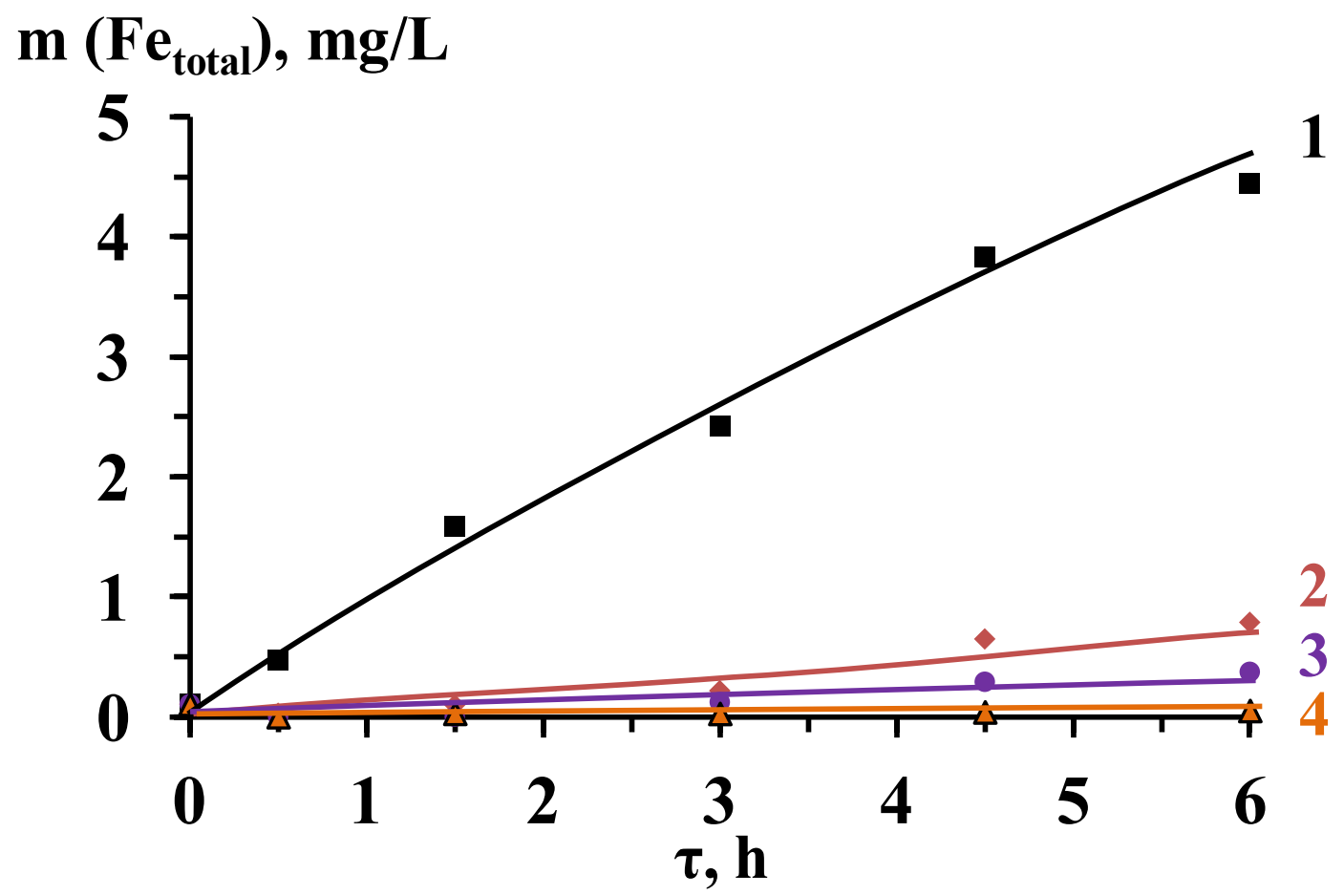

Figure 3. The total content of iron in the background electrolyte $(1)$ and in the presence of 50 (2), 500 (3) and $2000 \mathrm{mg} / \mathrm{L}$ of TBA (4).

There are several methods to calculate the contributions of the surface film component from the correlations presented above. In practice, it is more convenient to use " $g$ $\Delta m_{s p}\left(Z_{C P}\right)-\lg \tau$ " diagrams in the modified method, as it is described in [3], due to the low values of $\Delta m_{s p}$ at small test times, especially in inhibited environments. Since the described diagrams are an intermediate stage in the calculation and they are completely similar to the diagrams considered in [3], they are not presented in this paper. Then $Z_{C P}$ in inhibited environment is calculated from the diagrams obtained; $Z_{\text {total }}$ and $Z_{\text {in }}$ are calculated after $Z_{C P}$ [3].

The results of the calculations (Fig. 4) show that $Z_{C P}$ in inhibited $\mathrm{H}_{2} \mathrm{~S}$-containing liquid increases with time irrespective of the inhibitor content and the calculation method while, on the contrary, $Z_{\text {in }}$ decreases in all cases. However, the contribution values at the similar trend of changes strongly depend on $C_{i n}$. For example, $Z_{C P}$ starts to dominate at a small inhibitor concentration $(50 \mathrm{mg} / \mathrm{L})$ after 1 or 2 hours (depending on the calculation method) though $Z(T B A) \approx 75 \%$. Therefore, the corrosion product film at such inhibitor concentration has a greater contribution to the overall protective effect than the inhibitor itself. It is significant that $Z_{C P}$ values are almost identical at all calculation variants, whereas $Z_{i n}$ is approximately $30 \%$ higher in the original method [3] than in the modified ones.

The closest results according to all calculation procedures are observed in the range of medium inhibitor concentrations $(500 \mathrm{mg} / \mathrm{L}) . Z_{\text {in }}$ dominates during the first $3-5$ hours of the test, later a CP film makes the largest contribution to total protection. 
$\mathbf{Z}_{\mathbf{C P}}$
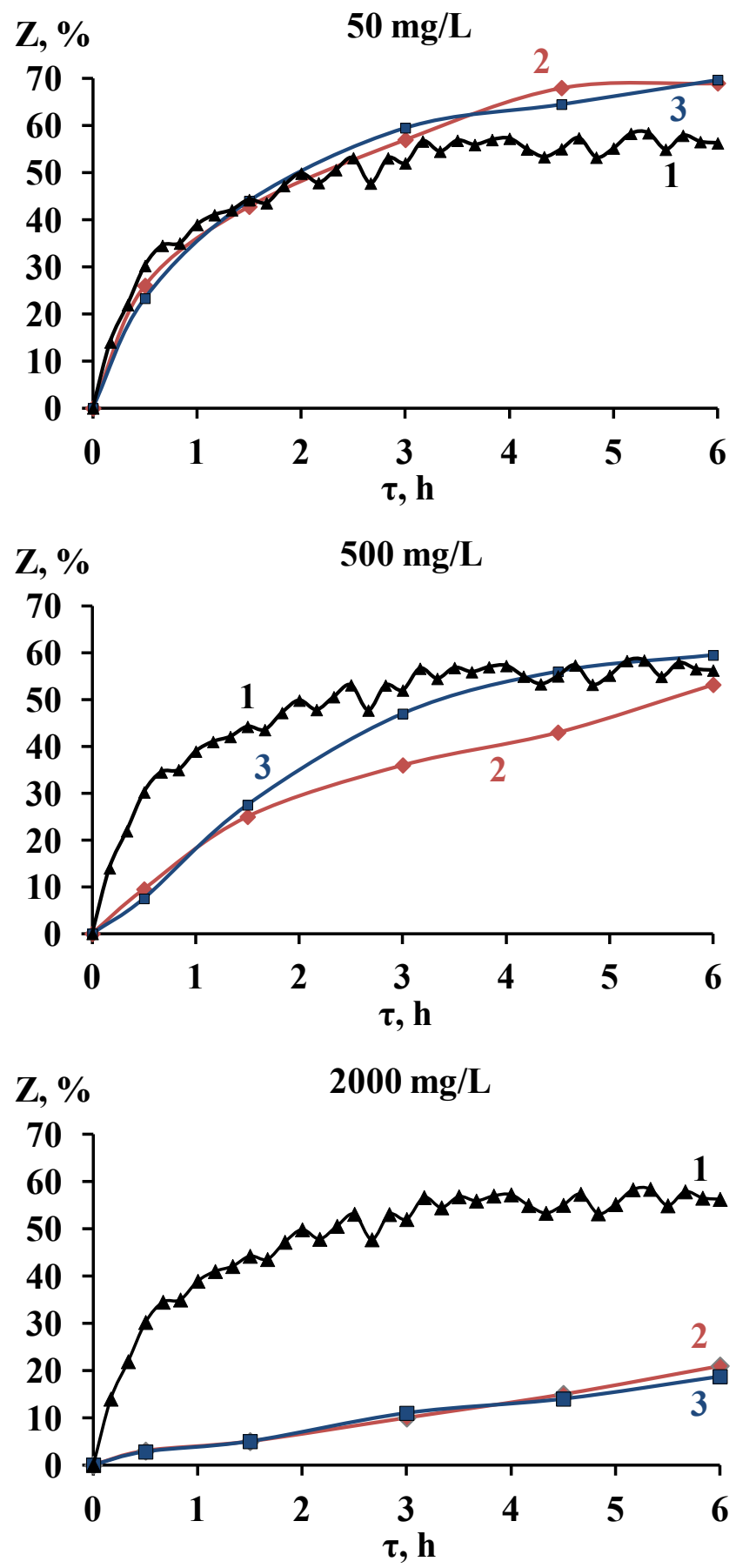

$\mathbf{Z}_{\text {in }}$
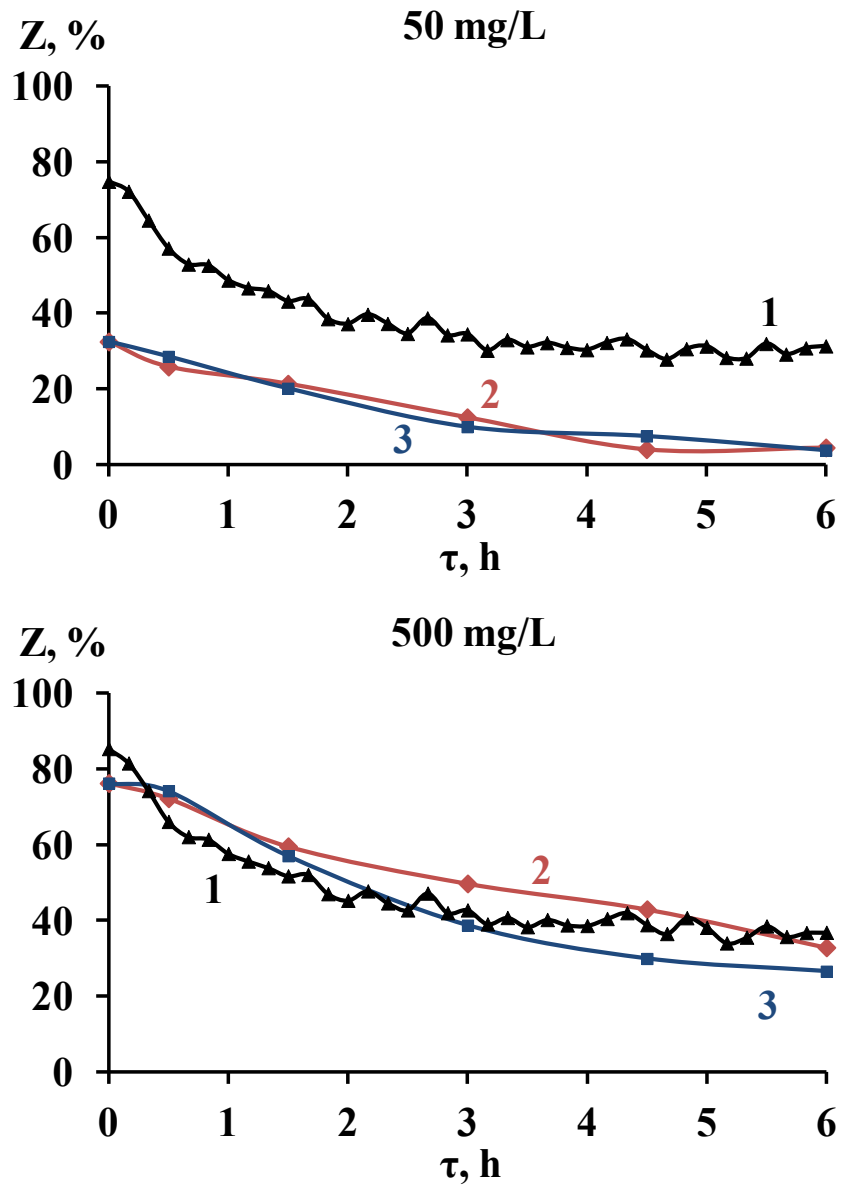

$2000 \mathrm{mg} / \mathrm{L}$

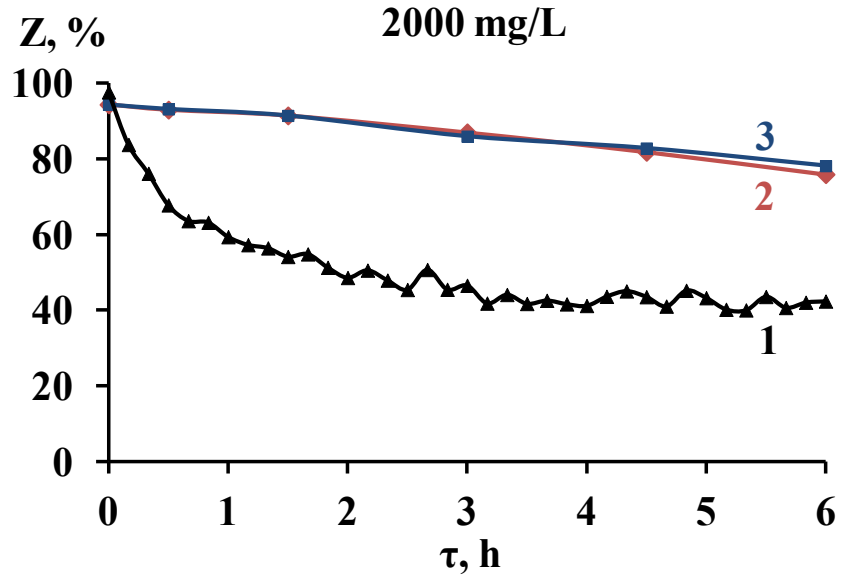

Figure 4. Contributions of $\mathrm{CP}$ (left) and inhibitor (right) films to the total protective effect calculated according to original (l) and modified methods without (2) and with dissolution of CP film taken into account (3), $C\left(\mathrm{H}_{2} \mathrm{~S}\right) \approx 3 \mathrm{~g} / \mathrm{L}$ (saturated liquid), $C(T B A)=50,500,2000$ $\mathrm{mg} / \mathrm{L}$.

The most interesting results are obtained at a high amine concentration $(2 \mathrm{~g} / \mathrm{L})$. In this case, the $Z_{C P}$ calculated by the original method is considerably greater, while on the contra- 
ry, $Z_{\text {in }}$ is smaller than similar values determined by the modified methods. As consequence, the original method testifies to the leading part of the CP film in $Z_{\text {total }}$, and the modified methods give an opposite estimate. According to the conclusions published in [3], an explanation for this distinction is that the original method that disregards the decrease in $\mathrm{CP}$ film thickness considerably overestimates its contribution to $Z_{\text {total }}$. Thus, it follows from the data obtained in the present work that the higher protective effect a corrosion inhibitor, the greater error of $Z_{C P}$ estimation.

It is necessary to notice that inhibitor and CP film contributions do not become constant (especially in the modified method) and, hence, while steel HSC will not completely stop, $Z_{C P}$ will gradually increase and $Z_{i n}$ will decrease. Finally the moment of their balance or even $Z_{C P}$ domination could come even in case of $C(T B A)=2 \mathrm{~g} / \mathrm{L}$.

It is important to mention that the results of the modified method practically do not change depending on whether dissolution of CP film is considered or not. Therefore, processes of CP film dissolution and HSC rate itself equally depend on the presence of a corrosion inhibitor in the environment. In other words, an inhibitor equally slows down both steel corrosion rate and dissolution of a polysulfide film. However, this conclusion based on the result of the present research is probably applicable only to HSC inhibition and an additional experimental check of the above mentioned conclusion is required for other corrosive environments.

\section{Conclusion}

1. A modification of the method for separate estimation of surface film components contributions to the total protective effect considering the thickness change of corrosion products film under the influence of inhibitor and dissolution of this film is offered. The results of the original and modified methods are compared by the example of steel HSC inhibition in liquid phase by tributylamine.

2. The error in estimation of corrosion product film contribution in the original method rises as the inhibitor protective effect increases. It is due to the initial assumption about equal protection effectiveness of corrosion product film in the background and inhibited environments.

3. The results of the modified method do not depend on whether corrosion product film dissolution is taken into consideration. Thus, tributylamine equally inhibits steel HSC and sulfide film dissolution.

\section{References}

1. R. V. Kashkovskiy, Proceedings of "Oil Gas Scientific Research Project", Institute of SOCAR, 2011, 2, no. 2, 48.

2. R. V. Kashkovskiy, Yu. I. Kuznetsov and R. K. Vagapov, European Corrosion Congress 2010, EUROCORR 2010, Moscow, 2010, 3546.

3. V. I. Vigdorovich, L. E. Tsygankova and N. V. Shel, Surface and Interface Analysis, 2010, 42, 626. 
4. R. V. Kashkovskiy, Yu. I. Kuznetsov and L. P. Kazansky, Corrosion Science, 2012, 64, 126.

5. R. V. Kashkovskiy and Yu. I. Kuznetsov, Korroz.: Mater. Zashch., 2013, 3, 20 (in Russian).

6. GOST (USSR Standart) 4011-72, Drinking water. Methods for determination of total iron (in Russian).

7. R. V. Kashkovskiy and Yu. I. Kuznetsov, Int. J. Corros. Scale Inhib., 2012, 1, 117. doi: $\underline{10.17675 / 2305-6894-2012-1-2-117-129}$ 